\title{
The effect of marrow stromal cells on TRAF6 expression levels in myeloma cells
}

\author{
HONGMING HUANG ${ }^{1}$, ZHONGWEI SUN $^{1}$, XUDONG WANG $^{2}$, XINXIN LIU ${ }^{1}$, \\ WENXIU NA ${ }^{1}$, RUIRONG XU ${ }^{1}$, RUNSHENG DING ${ }^{1}$ and HONG LIU ${ }^{1}$ \\ ${ }^{1}$ Department of Hematology and ${ }^{2}$ Surgical Comprehensive Laboratory, The Affiliated Hospital \\ of Nantong University, Nantong, Jiangsu 226001, P.R. China
}

Received July 17, 2015; Accepted January 6, 2017

DOI: $10.3892 / \mathrm{ol} .2017 .6322$

\begin{abstract}
Tumor necrosis factor receptor-associated factor 6 (TRAF6) is an important E3 ubiquitin ligase, which is key to immunity. TRAF6 has been implicated in the invasive growth and metastasis of various types of cancer, including squamous cell carcinoma, gastric cancer, myelodysplastic syndromes and acute myeloid leukemia. In the present study, associations between multiple myeloma (MM) and TRAF6, its downstream component nuclear factor- $\kappa \mathrm{B}(\mathrm{NF}-\kappa \mathrm{B})$ and bone marrow stromal cells (MSC) were investigated. The TRAF6 protein expression levels of 18 patients were positively correlated with the protein levels of $\beta-2$ microglobulin $\left(r^{2}=0.3472\right.$; $\mathrm{P}=0.01)$ and negatively correlated with albumin protein levels $\left(r^{2}=0.5494 ; \mathrm{P}=0.0004\right)$. In vitro expression of the TRAF6 protein, phosphorylated transcription factor p65 and phosphorylated p100 in myeloma cell lines was induced by MSCs from patients with MM. In addition, the in vitro expression of TRAF6 was associated with an enhanced proliferation rate of myeloma cells, which was blocked by silencing TRAF6 using small interfering RNA. Due to the association between the TRAF6-NF- $\mathrm{B}$ signaling pathway in myeloma cells and MSCs, this signaling pathway may be a useful prognostic and therapeutic target in myeloma.
\end{abstract}

\section{Introduction}

Tumor necrosis factor receptor-associated factor 6 (TRAF6) is an important E3 ubiquitin ligase (1) that is key to innate and adaptive immunity (2). TRAF6 has been reported to be involved in the invasive growth and metastasis of squamous

Correspondence to: Mrs. Hongming Huang or Mrs. Hong Liu, Department of Hematology, The Affiliated Hospital of Nantong University, 20 Xisi Road, Nantong, Jiangsu 226001, P.R. China

E-mail: hhmmmc@163.com

E-mail: hongliu63@126.com

Key words: multiple myeloma, marrow stromal cell, nuclear factor- $\kappa \mathrm{B}$, tumor necrosis factor receptor-associated factor 6 , microenvironment, prognosis cell carcinoma (3) and gastric cancer (4), as well as myelodysplastic syndromes and acute myeloid leukemia (5). Due to the importance of the numerous signal transduction pathways in which it is involved, TRAF6 may be a potential target for the treatment of cancer (6).

Multiple myeloma (MM) is associated with a dysregulated cell microenvironment, in which the marrow stromal cells (MSCs) promote the growth of myeloma cells (7). In osteoclasts, TRAF6 mediates signal transduction from the receptor activator of nuclear factor- $\kappa \mathrm{B}(\mathrm{NF}-\kappa \mathrm{B}) /$ receptor activator of $N F-\kappa B$ ligand (RANK/RANKL) signaling pathway (8); RANKL is primarily expressed in stromal cells (9). Inhibition of TRAF6 expression is able to suppress osteoclast proliferation and induce apoptosis (10). Furthermore, macrophages (11) and dendritic cells (12) in the microenvironment of myeloma cells express inflammatory factors, including TRAF6. The toll-like receptor (TLR)-TRAF6-NF- $\kappa \mathrm{B}$ signaling pathway may serve an important role in the development and progression of myeloma $(13,14)$.

However, the function of TRAF6 in myeloma cells has yet to be elucidated. Chen et al (15) demonstrated that TRAF6 knockdown using small interfering RNA (siRNA) molecules is able to inhibit the proliferation of myeloma cells and induce apoptosis, suggesting that TRAF6 may be a novel therapeutic target for myeloma. As TRAF6 is a signaling adapter molecule and patients with MM often overexpress various cytokines (16), the present study hypothesized that increased TRAF6 expression levels may be affected by stimulation of microenvironment and promote cell proliferation upon upstream signaling.

\section{Materials and methods}

Primarymyelomacells and human myeloma cell lines (HMCL). Bone marrow samples were obtained from 18 subjects with recently diagnosed $\mathrm{MM}$ and 3 healthy donors at The Affiliated Hospital of Nantong University (Nantong, China), from January 2014 to December 2014. Patient characteristics are presented in Table I.

Bone marrow mononuclear cells (BMMCs) were obtained using gradient centrifugation (716 x $g ; 20 \mathrm{~min}$; room temperature) in Ficoll ${ }^{\circledR}$-Paque Premium media (no. 17-5442-02; GE Healthcare Life Sciences, Uppsala, Sweden). Primary 
myeloma cells were purified using cluster of differentiation (CD) 138 microbeads, according to the manufacturer's protocol (Miltenyi Biotec GmbH, Bergisch Gladbach, Germany). The U266 and RPMI-8226 MM cell lines were purchased from the Beijing Cell Library of the Chinese Academy of Sciences (Beijing, China). The Ethics Committee of The Affiliated Hospital of Nantong University approved the study protocol. Written informed consent was obtained from all participants.

Bone marrow-derived MSCs. BMMCs were extracted from three patients and three healthy donors (HD), suspended in Dulbecco's modified Eagle's medium (DMEM; Gibco; Thermo Fisher Scientific, Inc., Waltham, MA, USA) supplemented with $10 \%$ fetal bovine serum (FBS; Gibco; Thermo Fisher Scientific, Inc.). Non-adherent cells were removed after a 24-h incubation at $37^{\circ} \mathrm{C}$ in an atmosphere containing $5 \% \mathrm{CO}_{2}$ and the medium was subsequently changed every 3-4 days. Upon reaching $>80 \%$ confluency, cells were detached using $0.125 \%$ trypsin and $0.01 \%$ EDTA and flow cytometry analysis (FACSAria II; BD Biosciences, Franklin Lakes, NJ, USA) was performed. Cells were subsequently split into new flasks and $\sim 1 \times 10^{5}$ cells from the fourth passage were resuspended in 24-well plates in $1 \mathrm{ml} \mathrm{DMEM}$ and cultured until $~ 80 \%$ confluency was reached.

Co-cultivation of HMCLs with MSCs. The U266 and RMPI-8226 cell lines were maintained in RPMI-1640 medium supplemented with $10 \%$ FBS, 2 mmol/l glutamine and $1 \%$ penicillin/streptomycin (all from Gibco; Thermo Fisher Scientific, Inc.). To evaluate the effect of MSCs obtained from MM patients on myeloma cells, the U266 and RMPI-8226 cell lines (including siRNA transfected cells) were washed in PBS twice, cultured at $37^{\circ} \mathrm{C}$ in an atmosphere containing $5 \% \mathrm{CO}_{2}$ in 24-well plates with RPMI-1640 media without FBS for $4 \mathrm{~h}$ and recollected. Approximately $1 \times 10^{6}$ cells were subsequently resuspended in 24-well plates containing MSCs $\left(6 \times 10^{5}\right.$ cells) with $>80 \%$ confluency.

Western blot analysis. Cells were lysed using $5 \mathrm{X}$ Laemmli sample buffer consisting of $50 \mathrm{mM}$ Tris buffer (pH 6.8), $2 \%$ sodium dodecyl sulfate and $10 \%$ glycerol, and containing the following protease inhibitors: Leupeptin $(1 \mu \mathrm{g} / \mathrm{ml})$, aprotinin $(1 \mu \mathrm{g} / \mathrm{ml})$ and $2 \mathrm{mM}$ phenylmethylsulfonyl fluoride (Sigma-Aldrich; Merck Millipore, Darmstadt, Germany). Whole cell lysates $(40 \mathrm{mg}$ ) were separated by $10 \%$ SDS-PAGE and transferred to polyvinylidene fluoride (PVDF) membranes (Bio-Rad Laboratories, Inc., Hercules, CA, USA). PVDF membranes were blocked using PBS with Tween-20 (PBST; $10 \mathrm{mM}$ Tris- $\mathrm{HCl}, \mathrm{pH}$ 7.5, $150 \mathrm{mM} \mathrm{NaCl}, 0.1 \%$ Tween-20) containing 5\% skimmed milk, and then incubated overnight at $4^{\circ} \mathrm{C}$ with antibodies against TRAF6 (dilution, 1:1,000; no. ab40675; Abcam, Cambridge, UK) or antibodies against p-p65 or p-p100 (dilution, 1:1,000; p-p65, no. 3033; p-p100, no. 4810; Cell Signaling Technology, Inc., Danvers, MA, USA). The PVDF membranes were washed three times in PBST and incubated with a peroxidase-conjugated AffiniPure Goat Anti-Rabbit Immunoglobulin G (H+L) (dilution, 1:5,000; no. 111-035-003; Jackson ImmunoResearch Laboratories, Inc., West Grove, PA, USA) for $2 \mathrm{~h}$ at room temperature. $\beta$-actin was detected using the above method but with the anti- $\beta$-actin
Table I. Clinical features of patients with $\mathrm{MM}$ and healthy donors.

A, Patients with MM.

Parameters

Patients with myeloma

Number

Median age (range), years

$57(37-72)$

Gender

Male

15

Female

3

IgG positive

IgA positive

IgD positive

$\kappa \mathrm{Fc}$ positive

$\lambda$ Fc positive

DS stage

I

II

III

Organ involved

Renal failure

Osteolysis

Mean number of plasma cells

$41.2(9-92)$

in the BM at diagnosis (\%)

B, Healthy donors.

\begin{tabular}{lc}
\hline Parameters & Healthy donors \\
\hline Number & 3 \\
Median age (range), years & $38(30-45)$ \\
Gender & \\
Male & 3 \\
Female & 0 \\
\hline
\end{tabular}

Fc, free chain; DS, Durie-Salmon; BM, bone marrow; Ig, immunoglobulin; MM, multiple myeloma.

antibody (dilution, 1:1,000; no. 4970; Cell Signaling Technology, Inc., Danvers, MA, USA). Following washing with PBST, the proteins were visualized using BioSpectrum ${ }^{\circledR}$ MultiSpectral Imaging System (Ultra-Violet Products Ltd., Cambridge, UK), and quantified using Quantity One 1-D Analysis software (version 4.6.9; Bio-Rad Laboratories, Inc.) and the developer 20X LumiGLO $^{\circledR}$ reagent and 20X peroxide, (no. 7003; Cell Signaling Technology, Inc.). Relative expression levels of the target protein were measured as the gray value ratio of target protein content to $\beta$-actin content.

Transfection of myeloma cell lines with TRAF6 siRNA. Prior to transfection, $6 \times 10^{5}$ cells were seeded in 6 -well plates in RPMI-1640 supplemented with $10 \% \mathrm{FBS}$ at $37^{\circ} \mathrm{C}$. The following day, $50 \mathrm{nM}$ of TRAF6 siRNA (Shanghai 
A
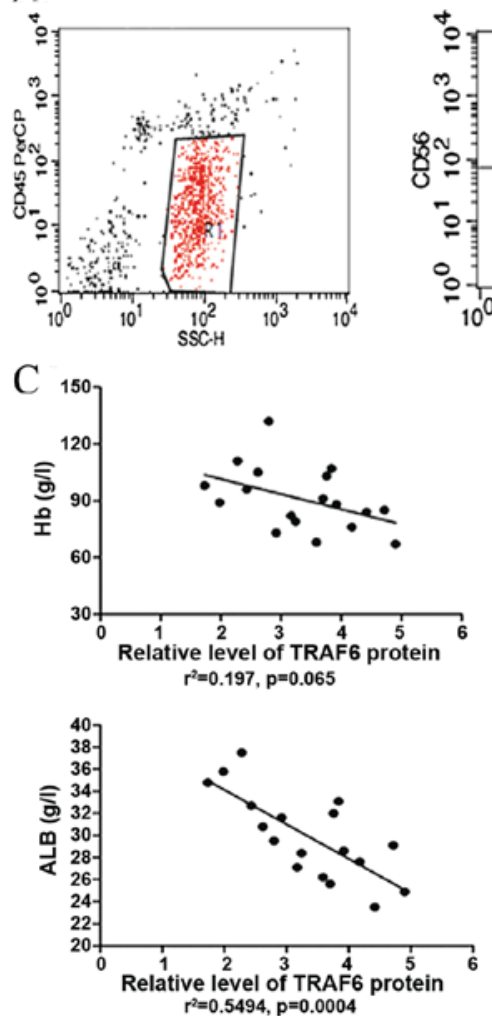
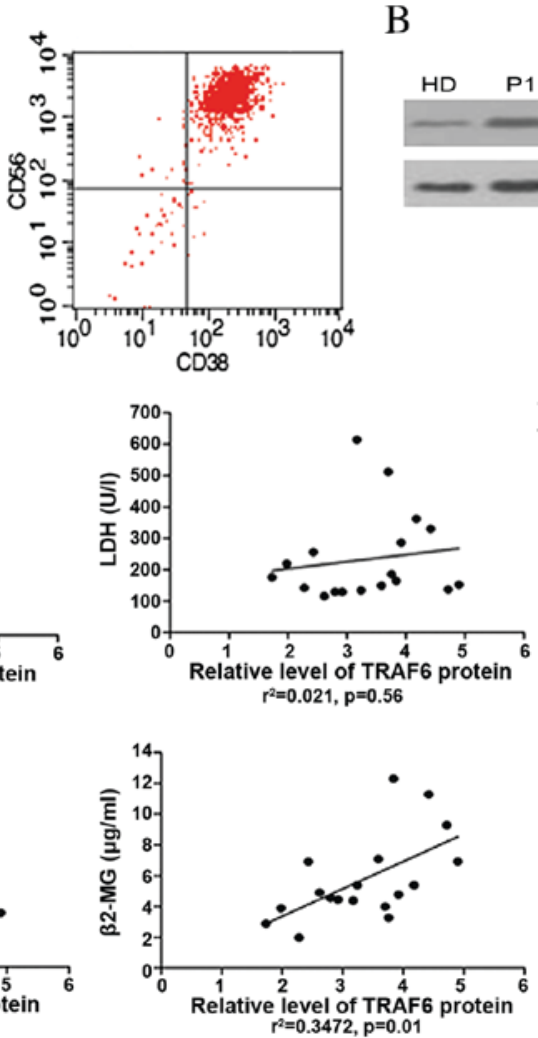

$\mathrm{B}$

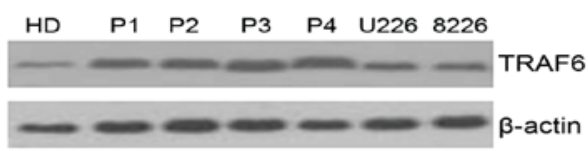

D

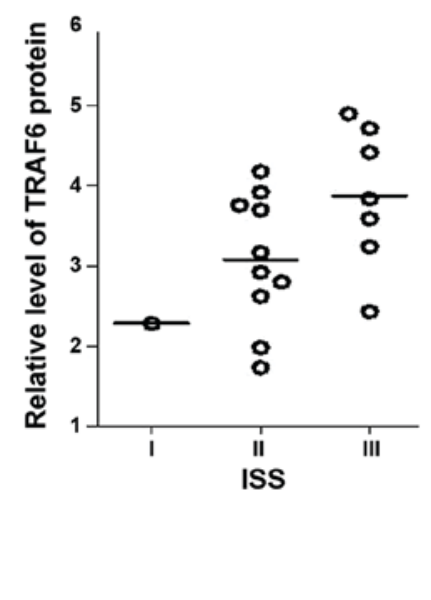

Figure 1. Expression of TRAF6 in primary myeloma cells and HMCLs. (A) Representative flow cytometry profile of purified CD138+ primary myeloma cells, presenting the primary myeloma cells $\left(\mathrm{C} 138^{+} \mathrm{CD} 56^{+} \mathrm{CD} 38^{+}\right)$were $>90 \%$ pure. (B) Western blot analysis of TRAF6 protein expression in myeloma cells. (C) Correlation between TRAF6 expression levels in primary myeloma cells from 18 patients with MM and various prognostic indices. (D) TRAF6 expression in plasma cells from patients with MM with various ISS classifications. TRAF6, tumor necrosis factor receptor-associated factor 6; CD, cluster of differentiation; $\mathrm{Hb}$, hemoglobin; ALB, albumin; LDH, lactate dehydrogenase; $\beta 2-\mathrm{MG}, \beta 2$ Microglobulin; ISS, International Scoring System; MM, multiple myeloma; HMCL, human myeloma cell line; HD, healthy donor; P1-4, patients 1-4.

GenePharma Co, Ltd., Shanghai, China) was added to each well with Entranster ${ }^{\mathrm{TM}}-\mathrm{R}$ (Engreen Biosystem Co., Ltd., Beijing, China) and cultured for a further $48 \mathrm{~h}$ at $37^{\circ} \mathrm{C}$ in an atmosphere containing $5 \% \mathrm{CO}_{2}$. RNA interference oligomers were complementary to the TRAF6 mRNA, and were as follows: Sense 5'-GCAGAUGGGGCAUUCAUATT-3'; antisense 5'-UAUGAAUGCCCCAUCUGCTT-3'). The negative control siRNAs were as follows: Sense 5'-UUCUCCGAACGU GUCACGUTT-3'; antisense 5'-ACGUGACACGUUCGGAGA ATT-3'. Scrambled siRNAs were used as controls (Shanghai GenePharma Co., Ltd.).

Cell proliferation assay. Transfected cells were seeded in 96 -well plates at a density of $5 \times 10^{3} /$ well, and cultured at $37^{\circ} \mathrm{C}$ in a humidified incubator for $48 \mathrm{~h}$. Subsequently, $10 \mu \mathrm{l}$ Cell Counting Kit-8 (CCK-8) solution (no. CK04; Dojindo Molecular Technologies, Inc., Kamamoto, Japan) was added to each well and the cells were incubated for a further $2 \mathrm{~h}$. Optical density (OD) values were determined at a wavelength of $450 \mathrm{~nm}$ using a microplate reader (Synergy HT; BioTek Instruments, Inc., Winooski, VT, USA).

Clinical correlation study. Blood samples were obtained from 18 patients (Table I) with MM on the second day following admission to The Affiliated Hospital of Nantong University. Blood levels of hemoglobin ( $\mathrm{Hb})$, lactate dehydrogenase $(\mathrm{LDH}), \beta 2 \mathrm{Microglobulin}(\beta 2 \mathrm{M})$ and albumin were evaluated in biochemical analysis department using standard clinical protocols.

Statistical analysis. SPSS version 19.0 (IBM SPSS, Armonk, NY, USA) was used for statistical analyses, and the results were presented as the mean \pm standard deviation. The Student's $t$-test was used to compare the expression levels of TRAF6 between the groups. $\mathrm{P}<0.05$ was considered to indicate a statistically significant difference.

\section{Results}

Expression levels of TRAF6 in myeloma cells are correlated with prognosis. BMMCs from 18 patients with MM were purified using CD138 microbeads, and CD56 and CD38 surface phenotypes were subsequently detected using standard clinical protocols. All purified primary myeloma cells $\left(\mathrm{C} 138^{+}\right.$ $\mathrm{CD} 6^{+} \mathrm{CD} 8^{+}$) were $>90 \%$ pure (Fig. $1 \mathrm{~A}$ ). Immunoblotting was used to determine TRAF6 protein levels in BMMCs obtained from patients, as well as in U266 and RPMI-8226 cells (Fig. 1B). The correlations between the expression levels of TRAF6 protein in primary myeloma cells and the $\mathrm{Hb}, \mathrm{LDH}$, $\beta 2 \mathrm{M}$ and albumin levels were examined. TRAF6 expression levels were not significantly correlated with the blood levels of $\mathrm{LDH}\left(\mathrm{r}^{2}=0.021 ; \mathrm{P}=0.56\right)$ or $\mathrm{Hb}\left(\mathrm{r}^{2}=0.197 ; \mathrm{P}=0.065\right)$ protein. However, the expression levels of TRAF6 were significantly and positively correlated with blood $\beta 2 \mathrm{M}$ levels $\left(r^{2}=0.3472\right.$; 
A
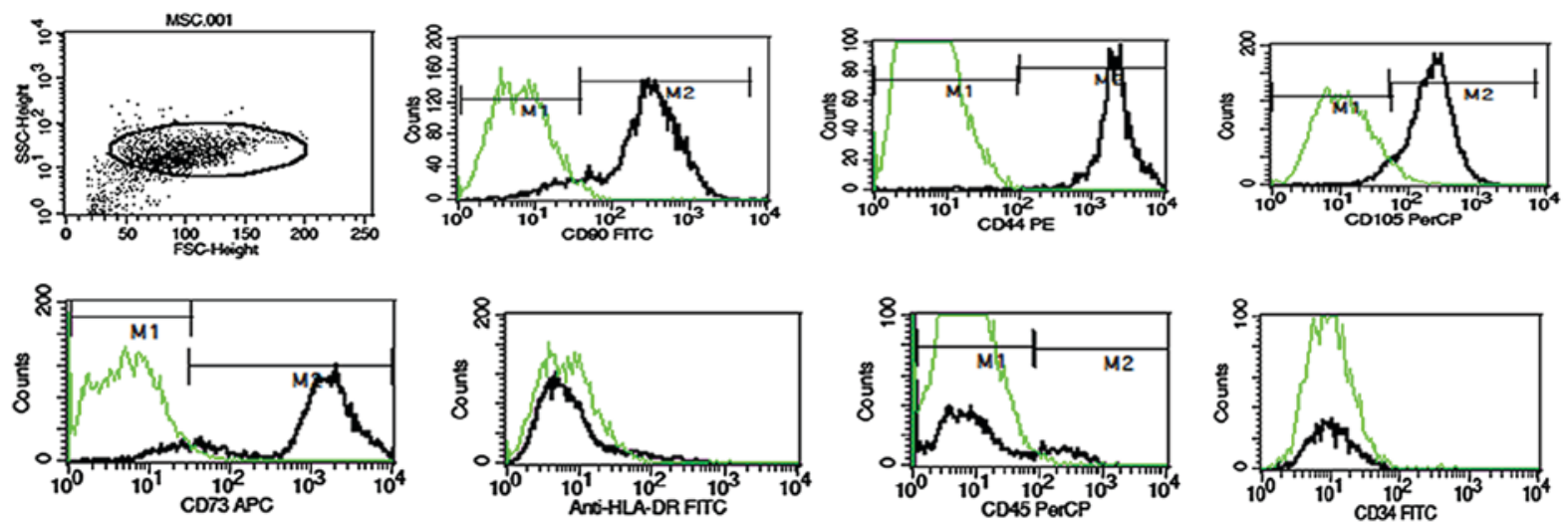

B

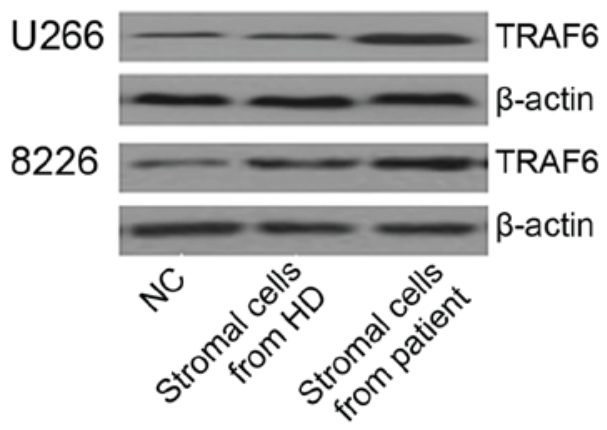

C

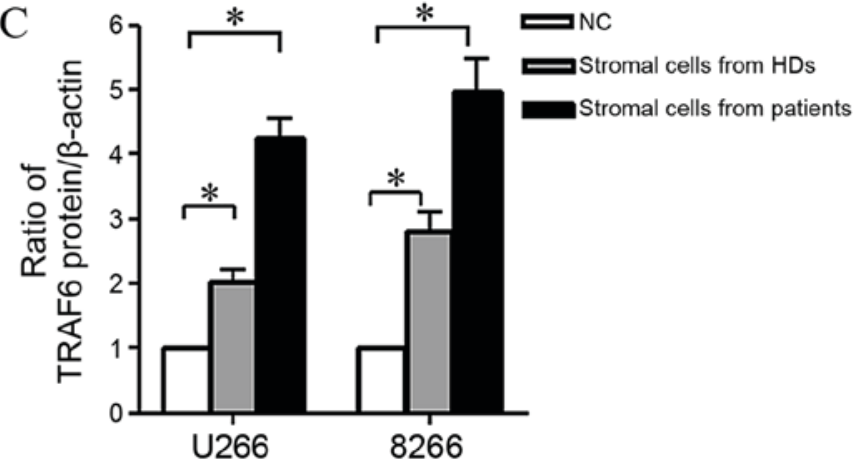

Figure 2. Bone marrow stromal cells induced TRAF6 expression in human myeloma cell lines. (A) Immunophenotypical characteristics of bone marrow stromal cells from a patient with myeloma. (B) Representative TRAF6 western blots. (C) Relative intensity of TRAF6 protein expression following co-culture with bone marrow stromal cells. The presented data are the mean \pm standard deviation of three experiments. * $\mathrm{P}<0.05$, as compared with cells cultured in Dulbecco's modified Eagle's medium. TRAF6, tumor necrosis factor receptor-associated factor 6; FSC, front scatter; SSC, side scatter; CD, cluster of differentiation; FITC, fluorescein isothiocyanate; PE, phycoerythrin; PerCP, peridinin chlorophyll protein; APC, allophycocyanin; HLA-DR, human leukocyte antigen-antigen D related; HD, healthy donor; MSC, bone marrow stromal cell.

$\mathrm{P}=0.01)$ and negatively correlated with albumin levels $\left(r^{2}=0.5494 ; \mathrm{P}=0.0004\right.$; Fig. $\left.1 \mathrm{C}\right)$. The International Staging System (ISS) of the International Myeloma Working Group (IMWG) was used to assess patients with MM (17) compared with the combination of the two objective prognostic variables, serum $\beta 2 \mathrm{M}$ and serum albumin, from this source. Although TRAF6 expression levels in myeloma cells tended to be higher in patients with MM at ISS stage III, compared with those at ISS stage II (Fig. 1D), no significant differences were observed, possibly due to the small sample size of patients.

Immunophenotypic characteristics of MSCs. The immunophenotype of MSCs from three patients was detected using flow cytometry, with all cell samples positive for CD90 $(85.75 \pm 2.35 \%)$, CD44 (96.53 $\pm 3.79 \%)$, CD73 $(90.21 \pm 1.09 \%)$ and CD105 $(94.46 \pm 3.59 \%)$ expression, but negative for CD45, CD34 and human leukocyte antigen-antigen D related (HLA-DR) expression (Fig. 2A). Concordant with the results of previous study (18), the immunophenotype of the MSCs from the three HDs was similar to that of the patients with MM, expressing CD90 (84.23 $\pm 3.85 \%)$, CD44 (96.76 $\pm 3.9 \%)$, CD73 (91.67 $\pm 2.36 \%)$ and CD105 (95.15 $\pm 2.76 \%)$, and $\leq 5 \%$ of MSCs expressing CD45, CD34 and HLA-DR (data not shown).

TRAF6 expression is enhanced in myeloma cells by MSCs. U266 and RPMI-8226 cells were serum-starved for $4 \mathrm{~h}$ and then cultured in DMEM containing MSCs from a patient with MM and a HD, or without the stromal cells [(negative control (NC)] for $48 \mathrm{~h}$. TRAF6 protein expression levels in HMCLs were subsequently evaluated. As compared with NCs, the expression levels of TRAF6 protein were higher in U266 and RPMI-8226 cells co-cultured with stromal cells from a HD; however, the highest TRAF6 expression levels were observed in cells co-cultured with MSCs from a patient with MM (Fig. 2B). These experiments were performed with three myeloma patients and three HDs, and the mean and standard deviation were derived from the experiments in which the gray value ratios were evaluated three times. The data revealed that TRAF6 protein levels in myeloma cells increased significantly when co-cultured with HD stromal cells or MM patient stromal cells, as compared with NCs ( $<<0.05$; Fig. 2C).

Effects of TRAF6 silencing on myeloma cell proliferation when co-cultured with MSCs. The transfection efficiency of $50 \mathrm{nmol} / \mathrm{l}$ siTRAF6 was 92 and $87 \%$ in U266 and RPMI-8226 cells, respectively, as determined by fluorescent microscopy after $48 \mathrm{~h}$ of incubation (Fig. 3A). Following transfection, TRAF6 protein levels decreased after $48 \mathrm{~h}$ incubation (Fig. 3B), suggesting effective siTRAF6 transfection in myeloma cells. To determine the viability of myeloma cells following siTRAF6 transfection, the cells were recollected, washed and co-cultured with patient stromal cells for 
A

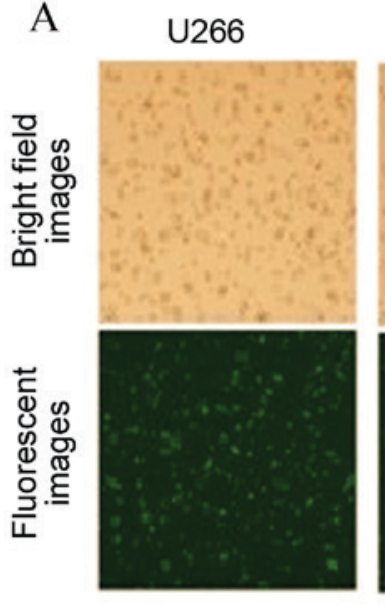

8226

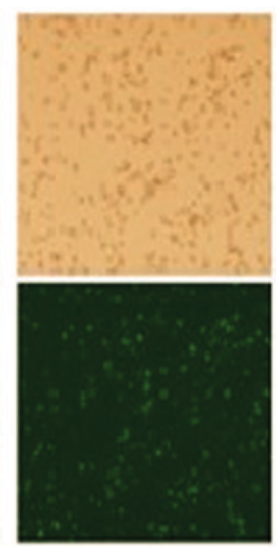

B

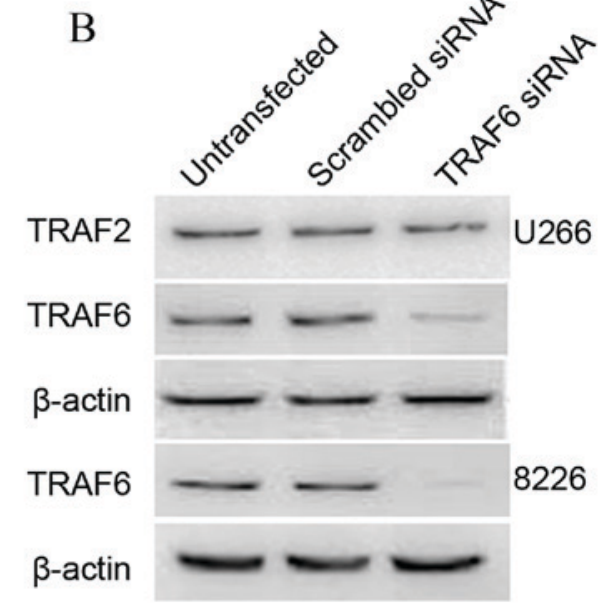

C
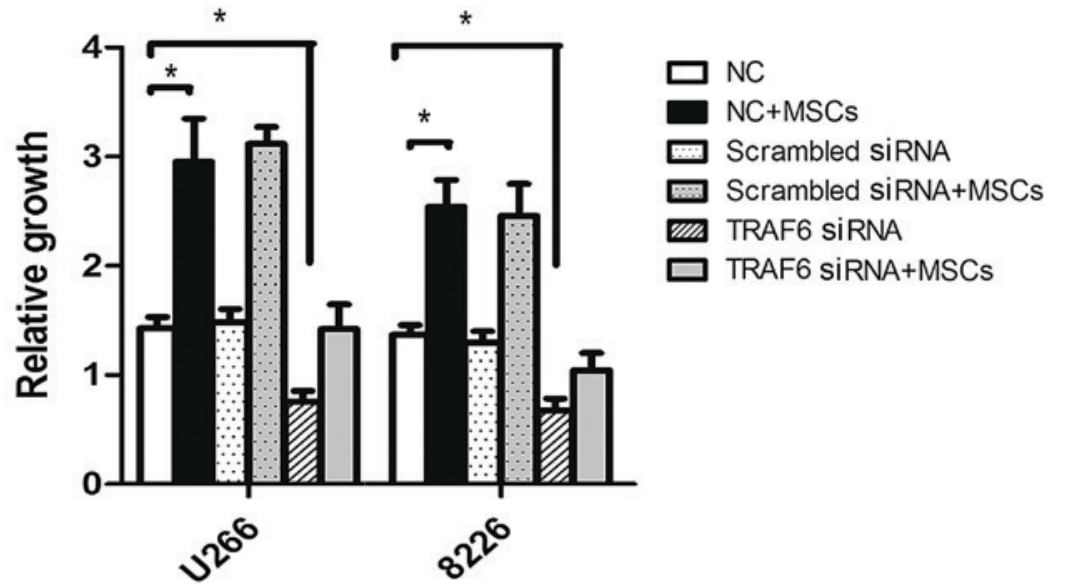

Figure 3. Effects of siRNA-mediated TRAF6 silencing on the growth of MM cells following co-culture with patient stromal cells. (A) Transfection efficiency of TRAF6 siRNA (50 nmol/l) in myeloma cell lines as detected by fluorescence microscopy (100x). (B) Western blot analysis of TRAF6 protein expression levels following transfection with TRAF6 siRNA. (C) TRAF6 siRNA significantly inhibited the proliferation of MM cell lines was significantly inhibited by TRAF6 siRNA, and significantly promoted cell proliferation following co-culture with patient stromal cells ("P<0.05, compared with NC). siRNA, small interfering RNA; TRAF6, tumor necrosis factor receptor-associated factor 6; NC, negative control; MSC, bone marrow stromal cell; MM, multiple myeloma.

48 h. The siTRAF6-transfected cells exhibited significantly decreased OD values, compared with control myeloma cells $(\mathrm{P}<0.05)$. HMCL cell growth increased significantly following co-culture with stromal cells from patients with $\mathrm{MM}(\mathrm{P}<0.05$, whereas no significant growth differences were observed in the TRAF6-silencing group following co-culture with patient stromal cells (Fig. 3C).

Effects of TRAF6 silencing on $N F-\kappa B$ protein levels in myeloma cells. A total of $48 \mathrm{~h}$ following siTRAF6 transfection, HMCLs were co-cultured with MSCs for a further $24 \mathrm{~h}$ prior to collection and the evaluation of p-p65 and p-p100 expression levels. All three HMCLs exhibited increased expression of p-p65 and p-p100 in response to co-culture with patient MSCs. By contrast, decreased expression levels of p-p65 and p-p100 were observed in TRAF6-silenced myeloma cells, even upon stimulation with patient stromal cells (Fig. 4A). Following co-culture with patient stromal cells, the siTRAF6-transfected cells exhibited significantly decreased expression levels of p-p65 and p-p100 $(\mathrm{P}<0.05)$, compared with non-siTRAF6-transfected cells (Fig. 4B). The levels of total p65, p100 and p52 expression in myeloma cells were not observed to be altered following co-culture with MSCs (Fig. 4C).

\section{Discussion}

A previous study demonstrated that increased expression levels of TRAF6 are present in myeloma cell lines and primary myeloma cells, and that TRAF6 siRNAs are able to decrease the downstream activity of NF- $\mathrm{BB}$ (19). This suggests that TRAF6 may be a novel target for the treatment of MM.

It has been established that TRAF6 is important in the osteoclasts of patients with MM (10); however, the role of TRAF6 in myeloma cells has yet to be elucidated. As TRAF6 is a signaling adapter molecule and patients with MM frequently overexpress various cytokines (16), it was hypothesized in the present study that TRAF6 expression may be associated with the tumor microenvironment (20) and promote cell proliferation upon upstream signal transduction.

The present study revealed that MM cell lines and CD138 ${ }^{+}$cells from patients with MM exhibited enhanced TRAF6 expression levels, and TRAF6 expression in the majority of patients with MM was higher, compared with that observed in MM cell lines. In the absence of cytokine stimulation, U266 and RPMI-8226 cell lines expressed TRAF6 autonomously. In addition, the presence of higher TRAF6 expression levels in primary MM cells suggests that TRAF6 expression in MM cells may be regulated by the 
A

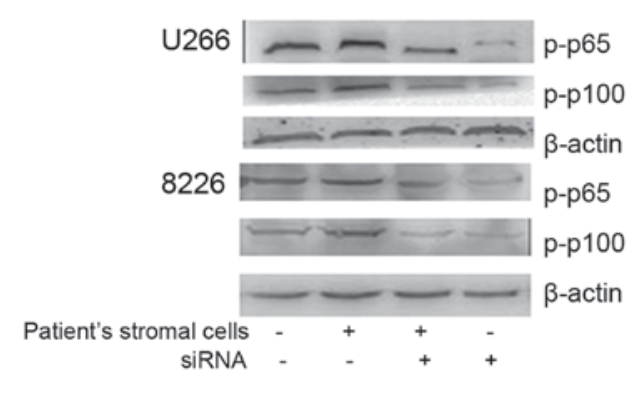

$\mathrm{C}$

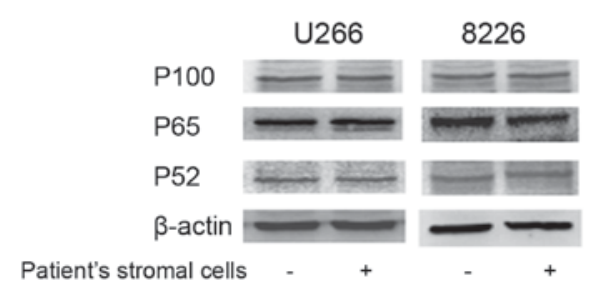

B
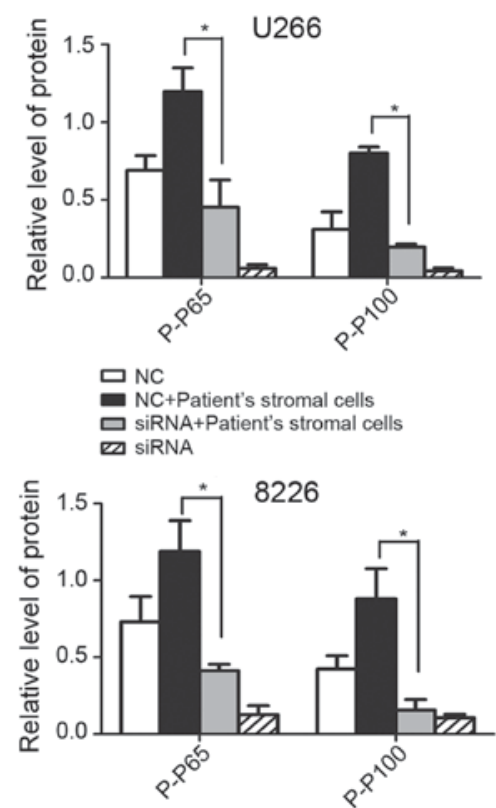

Figure 4. Effects of TRAF6 silencing on the expression levels of p-p65 and p-p100 protein following the addition of patient stromal cells. (A) The TRAF6 siRNA-transfected U266 and RPMI8226 myeloma cells were co-cultured with patient stromal cells for 24 h prior to immunoblotting with anti-p-p65 and anti-p-p100. (B) The two TRAF6 siRNA-transfected cell lines exhibited significantly decreased expression levels of p-p65 and p-p100, compared with non-transfected cells $\left({ }^{*} \mathrm{P}<0.05\right)$. (C) The U266 and RPMI8226 cells were co-cultured with patient stromal cells for $24 \mathrm{~h}$ prior to immunoblotting with anti-p65, anti-p100 and anti-p52. siRNA, small interfering RNA; TRAF6, tumor necrosis factor receptor-associated factor 6; p52, tumor protein 52; p-p65, phospho-p65; P-100, phospho-100; NC, negative control.

microenvironment and may be associated with the patient condition.

It has been established that the levels of biochemical markers, including $\beta 2 \mathrm{M}$, albumin and $\mathrm{LDH}$, are correlated with the pathogenesis of MM $(21,22)$. $\beta 2 \mathrm{M}$ levels are an independent prognostic factor, albumin levels are associated with tumor mass and physical status, LDH is one of the markers associated with disease stage and $\mathrm{Hb}$ levels are an important criterion for Durie-Salmon staging (23). In the present study, TRAF6 expression was not associated with the blood levels of $\mathrm{LDH}$ and $\mathrm{Hb}$, but was positively correlated with blood $\beta 2 \mathrm{M}$ levels and negatively correlated with blood albumin levels. The ISS for MM evaluates the levels of $\beta 2 \mathrm{M}$ and albumin (24); therefore, TRAF6 levels may correlate with patient prognosis. Based on the observation that TRAF6 expression is correlated with certain myeloma markers, it is hypothesized in the current study that TRAF6 may be a cell-survival factor that promotes the proliferation of myeloma cells, and its expression may controlled by upstream signaling occurring outside of myeloma cells.

To evaluate this hypothesis, the present study co-cultured MM cell lines with stromal cells from patients with myeloma, revealing TRAF6 expression was induced. Subsequently, siRNAs targeting the TRAF6 C-terminus were transfected into the myeloma cell lines and cell proliferation was evaluated. In concordance with previous studies, the stromal cells were able to stimulate the proliferation of myeloma cells $(25,26)$. In addition, cells transfected with TRAF6 siRNA exhibited growth inhibition, as well as partial resistance to the growth-stimulatory effects of stromal cells. The effects of the NF- $\mathrm{BB}$ family in myeloma cell proliferation have previously been established (27), during which the canonical p50/p65/inhibitor of $\kappa \mathrm{B}$ signaling pathway and the non-canonical $\mathrm{p} 52 / \mathrm{NF}-\kappa \mathrm{B}$-inducing kinase signaling pathway (including p100 of which p52 is a derivative) are activated to regulate the expression levels of certain downstream genes. In the present study, the expression of p-p65 and p-p100 was induced in response to patient stromal cells, indicating that the $\mathrm{NF}-\kappa \mathrm{B}$ family members may be activated by MSCs. In myeloma cells transfected with TRAF6 siRNA, the expression of p-p65 and p-p100 was downregulated, further supporting the hypothesis that myeloma stromal cells may secrete numerous cytokines that are able to regulate the transcriptional activity of NF- $\kappa \mathrm{B}$. The observation that TRAF6 siRNA is able to inhibit the proliferation of myeloma cells suggests that TRAF6 has an important role in signal transduction, possibly contributing to the proliferation of myeloma cells. Additionally, according to the aforementioned results, certain cytokines that are secreted from MSCs may enhance TRAF6 expression levels in myeloma cells and affect downstream targets in the NF- $\kappa$ B family.

Previous studies have demonstrated that CD40L, interleukin (IL)-1 (1), IL-17 (28) and RANKL (29) are able to bind to TRAF6 receptors, a number of which originate from stromal cells. Investigating the upstream signaling factors involved in this process may enable improved understanding of the TRAF6 signaling pathway in myeloma cells. As the TRAF6 signaling pathway is important in various other cell types, in addition to the microenvironment of myeloma cells (30), TRAF6 may be a novel target for the treatment of MM.

The interactions of the myeloma microenvironment, including osteoclasts, with myeloma cells and TRAF6 are essential to the functions of osteoclasts. Therefore, an 
investigation of TRAF6 and its upstream signaling pathway may aid the elucidation of novel therapeutic targets for myeloma, improving the available treatment strategies for myeloma.

Whilst the present study demonstrated that patient stromal cells are able to induce TRAF6 expression in myeloma cells, the cytokines and mechanisms underlying this process remain to be elucidated and must be investigated in future studies. In conclusion, the present study revealed that MSCs from patients with $\mathrm{MM}$ are able to stimulate the growth of myeloma cells via the TRAF6 signaling pathway, and that TRAF6 expression levels are correlated with patient prognosis. It is hypothesized that further investigation of the TRAF6 signal transduction pathway may improve understanding of the pathogenesis of MM and lead to the identification of novel therapeutic targets upstream of TRAF6.

\section{Acknowledgements}

The authors would like to thank the patients, clinical staff and their colleagues at The Affiliated Hospital of Nantong University (Nantong, China) for their assistance throughout the current study. This study was supported by the Young Scientists Fund of the National Natural Science Foundation of China (grant no. 81201857) and the Natural Science Foundation of Jiangsu Province, China (grant no. BK2011388).

\section{References}

1. Wu H and Arron JR: TRAF6, a molecular bridge spanning adaptive immunity, innate immunity and osteoimmunology. Bioessays 25: 1096-1105, 2003.

2. Zhong L, Cao F and You Q: Effect of TRAF6 on the biological behavior of human lung adenocarcinoma cell. Tumour Biol 34: 231-239, 2013.

3. Chaudhry SI, Hooper S, Nye E, Williamson P, Harrington K and Sahai E: Autocrine IL-1 $\beta$-TRAF6 signalling promotes squamous cell carcinoma invasion through paracrine $\mathrm{TNF} \alpha$ signalling to carcinoma-associated fibroblasts. Oncogene 32: 747-758, 2013.

4. Sun YS, Ye ZY, Qian ZY, Xu XD and Hu JF: Expression of TRAF6 and ubiquitin mRNA in skeletal muscle of gastric cancer patients. J Exp Clin Cancer Res 31: 81, 2012.

5. Fang J, Rhyasen G, Bolanos L, Rasch C, Varney M, Wunderlich M, Goyama S, Jansen G, Cloos J, Rigolino C, et al: Cytotoxic effects of bortezomib in myelodysplastic syndrome/acute myeloid leukemia depend on autophagy-mediated lysosomal degradation of TRAF6 and repression of PSMA1. Blood 120: 858-867, 2012.

6. Wang G, Gao Y, Li L, Jin G, Cai Z, Chao JI and Lin HK K63-linked ubiquitination in kinase activation and cancer. Front Oncol 2: 5, 2012 .

7. Abe M: Targeting the interplay between myeloma cells and the bone marrow microenvironment in myeloma. Int J Hematol 94 334-343, 2011

8. Armstrong AP, Tometsko ME, Glaccum M, Sutherland CL, Cosman D and Dougall WC: A RANK/TRAF6-dependent signal transduction pathway is essential for osteoclast cytoskeletal organization and resorptive function. J Biol Chem 277: 44347-44356, 2002.

9. Sezer O, Heider U, Zavrski I, Kühne CA and Hofbauer LC: RANK ligand and osteoprotegerin in myeloma bone disease. Blood 101: 2094-2098, 2003.

10. Hongming $\mathrm{H}$ and Jian $\mathrm{H}$ : Bortezomib inhibits maturation and function of osteoclasts from PBMCs of patients with multiple myeloma by downregulating TRAF6. Leuk Res 33: 115-122, 2009.

11. Zheng Y, Cai Z, Wang S, Zhang X, Qian J, Hong S, Li H, Wang M, Yang J and Yi Q: Macrophages are an abundant component of myeloma microenvironment and protect myeloma cells from chemotherapy drug-induced apoptosis. Blood 114: 3625-3628, 2009.
12. Tucci M, Stucci S, Strippoli S, Dammacco F and Silvestris F: Dendritic cells and malignant plasma cells: An alliance in multiple myeloma tumor progression? Oncologist 16: 1040-1048, 2011.

13. Wu Y, Zhu X, Li N, Chen T, Yang M, Yao M, Liu X, Jin B, Wang $\mathrm{X}$ and Cao X: CMRF-35-like molecule 3 preferentially promotes TLR9-triggered proinflammatory cytokine production in macrophages by enhancing TNF receptor-associated factor 6 ubiquitination. J Immunol 187: 4881-4889, 2011.

14. Kobayashi T, Walsh PT, Walsh MC, Speirs KM, Chiffoleau E, King CG, Hancock WW, Caamano JH, Hunter CA, Scott P, et al: TRAF6 is a critical factor for dendritic cell maturation and development. Immunity 19: 353-363, 2003.

15. Chen H, Li M, Campbell RA, Burkhardt K, Zhu D, Li SG, Lee HJ, Wang C, Zeng Z, Gordon MS, et al: Interference with nuclear factor kappa B and c-Jun NH2-terminal kinase signaling by TRAF6C small interfering RNA inhibits myeloma cell proliferation and enhances apoptosis. Oncogene 25: 6520-6527, 2006.

16. Zheng MM, Zhang Z, Bemis K, Belch AR, Pilarski LM, Shively JE and Kirshner J: The systemic cytokine environment is permanently altered in multiple myeloma. PLoS One 8: e58504, 2013.

17. Greipp PR, San Miguel J, Dune BG, Crowley JJ, Barlogie B, Bladé J, Boccadoro M, Child JA, Avet-Loiseau H, Kyle RA, et al: International staging system for multiple myeloma. J Clin Oncol 23: 3412-3420, 2005.

18. Hao M, Xie ZQ, Han YJ, An G, Meng HX, Huang J, Li CH, Zou DH and Qiu LG: Effect of mesenchymal stem cells on multiple myeloma cells growth and inhibition of bortezomib induced cell apoptosis. Zhonghua Xue Ye Xue Za Zhi 31: 680-683, 2010 (In Chinese).

19. Huang HM, Wang XF, Liu XX, Xu RR, Shi W, Ding RS and Jiang SH: Effects of down-regulated TRAF6 gene expression on the proliferation and apoptosis in multiple myeloma cells. Zhonghua Xue Ye Xue Za Zhi 34: 941-945, 2013 (In Chinese).

20. Liu H, Tamashiro S, Baritaki S, Penichet M, Yu Y, Chen H, Berenson $\mathrm{J}$ and Bonavida B: TRAF6 activation in multiple myeloma: A potential therapeutic target. Clin Lymphoma Myeloma Leuk 12: 155-163, 2012.

21. Kaneko M, Kanda Y, Oshima K, Nannya Y, Suguro M, Yamamoto R, Chizuka A, Hamaki T, Matsuyama T, Takezako N, et al: Simple prognostic model for patients with multiple myeloma: A single-cencer study in Japan. Ann Hematol 81: 33-36, 2002.

22. Dimopoulos MA, Barlogie B, Smith TL and Alexanian R: High serum lactate dehydrogenase level as a marker for drug resistance and short survival in multiple myeloma. Ann Intern Med 115: 931-935, 1991.

23. Nagura E: Prognositic factors in multiple myeloma. Nihon Rinsho 65: 2351-2356, 2007 (In Japanese).

24. Rajkumar SV and Kyle RA: Multiple myeloma: Diagnosis and treatment. Mayo Clin Proc 80, 1371-1382, 2005.

25. Gunn WG, Conley A, Deininger L, Olson SD, Prockop DJ and Gregory CA: A crosstalk between myeloma cells and marrow stromal cells stimulates production of DKK1 and interleukin-6: A potential role in the development of lytic bone disease and tumor progression in multiple myeloma. Stem Cells 24: 986-991, 2006.

26. Xu G, Liu K, Anderson J, Patrene K, Lentzsch S, Roodman GD and Ouyang H: Expression of XBP1s in bone marrow stromal cells is critical for myeloma cell growth and osteocast formation. Blood 119: 4205-4214, 2012.

27. Annunziata CM, Davis RE, Demchenko Y, Bellamy W, Gabrea A, Zhan F, Lenz G, Hanamura I, Wright G, Xiao W, et al: Frequent engagement of the classical and alternative NF-kappaB pathways by diverse genetic abnormalities in multiple myeloma. Cancer Cell 12: 115-130, 2007.

28. Rong Z, Cheng L, Ren Y, Li Z, Li Y, Li X, Li H, Fu XY and Chang Z: Interleukin-17F signaling requires ubiquitination of interleukin-17 receptor via TRAF6. Cell Signal 19: 1514-1520, 2007.

29. Theoleyre S, Wittrant Y, Tat SK, Fortun Y, Redini F and Heymann D: The molecular triad OPG/RANK/RANKL: Involvement in the orchestration of pathophysiological bone remodeling. Cytokine Growth Factor Rev 15: 457-475, 2004.

30. Liu H, Tamashiro S, Baritaki S, Penichet M, Yu Y, Chen H, Berenson $\mathrm{J}$ and Bonavida B: TRAF6 activation in multiple myeloma: A potential therapeutic target. Clin Lymphoma Myeloma Leuk 12: 155-163, 2012. 